REVISTA MATEMATICA de la

Universidad Complutense de Madrid

Volumen 5 , números 2 y 3 ; $[992$.

http://dx.doi.org/10.5209/rev_REMA.1992.v5.n2.17909

\title{
On Slice Knots in the Complex Projective Plane
}

\author{
AKIRA YASUHARA
}

\begin{abstract}
We investigate the knots in the boundary of the punctured complex projective plane. Our result gives an affirmative answer to a question raised by Suzuki. As an application, we answer to a question by Mathieu.
\end{abstract}

\section{INTRODUCTION}

Throughout this paper, we work in the smooth category, all manifolds are oriented and all the homology groups are with integral coefficients.

Let $M$ be a closed 4-manifold, $B^{4}$ an embedded 4-ball in $M$, and $K$ a knot in $\partial\left(M-\operatorname{Int} B^{4}\right)$. If $K$ bounds a properly embedded 2-disk in $M-\operatorname{Int} B^{4}$ then we call the knot $K$ a slice knot in $M$. Let Slice $(M)$ be the set of slice knots in $M$. It is well-known that Slice $\left(S^{4}\right)$ is proper subset of the set of knots (Fox and Milnor [3]) and Slice $\left(S^{4}\right)$ is a subset of Slice $(M)$. In [17], Suzuki proved that Slice $\left(S^{2} \times S^{2}\right)$ is equal to the set of knots, and asked the following question.

Question 1. Is there a 4-manifold $M$ such that Slice $\left(S^{4}\right)$ is a proper subset of Slice (M) and Slice (M) is a proper subset of the set of knots?

In [20], the author has proved that Slice $\left(C P^{2}\right)$ does not contain a $(-2,15)-$ torus knot. This assertion gives an affirmative answer to Question 1 since Slice $\left(S^{4}\right)$ is a proper subset of Slice $\left(C P^{2}\right)$ (Kervaire and Milnor [6]). In [20], the author could not find a knot that belongs to neither Slice $\left(C P^{2}\right)$ nor Slice $\left(\overline{C P^{2}}\right)$. In Section 2, we show that there exist the knots that belongs to neither Slice $\left(C P^{2}\right)$ nor Slice $\left(\overline{C P^{2}}\right)$.

1991 Mathematics Subject Classification: 57M25; 57M13, 57R95.

Editorial Complutense. Madrid, 1992. 
Let $K$ be a knot in $\partial\left(n_{1} C P^{2} \# n_{2} \overline{C P^{2}}-\right.$ Int $\left.B^{4}\right)$. The knot $K$ is an evenly slice knot in $n_{1} C P^{2} \# n_{2} \overline{C P^{2}}$ if $K$ bounds a properly embedded 2-disk in $n_{1} C P^{2} \# n_{2} \overline{C P^{2}}-$ Int $B^{4}$ that represents an element $z\left(\varepsilon_{1} \gamma_{1}+\ldots+\varepsilon_{n_{1}} \gamma_{n_{1}}+\right.$ $\left.\bar{\varepsilon}_{1} \bar{\gamma}_{1}+\ldots+\bar{\varepsilon}_{n_{2}} \bar{\gamma}_{n_{2}}\right)$ in $H_{2}\left(n_{1} C P^{2} \# n_{2} \overline{C P^{2}}-\right.$ Int $\left.B^{4}, \partial\right)$, where $\gamma_{1}, \ldots, \gamma_{n_{1}}, \bar{\gamma}_{1}, \ldots, \bar{\gamma}_{n_{2}}$ are standard generators of $H_{2}\left(n_{1} C P^{2} \# n_{2} \overline{C P^{2}}-\right.$ Int $\left.B^{4}, \partial\right), \varepsilon_{i}= \pm 1, \bar{\varepsilon}_{j}= \pm 1$ and $z$ is an integer. Let $e$-Slice $\left(n_{1} C P^{2} \# n_{2} \overline{C P^{2}}\right)$ be the set of evenly slice knots in $n_{1} C P^{2} \# n_{2} \overline{C P^{2}}$. (Note that e-Slice $\left(C P^{2}\right)=$ Slice $\left(C P^{2}\right)$ and e-Slice $\left.\overline{\left(C P^{2}\right.}\right)=$ Slice $\left(\overline{C P^{2}}\right)$.) In Section 3, we deal with in the case $n_{1}=n_{2}=1$ or $n_{1}=0$.

Let $K_{0}$ be a knot and $D^{2}$ a 2-disk intersecting transversely $K_{0}$ with the linking number $l k\left(\partial D^{2}, K_{0}\right)=l$. Let $p$ be a positive integer and $\varepsilon= \pm 1$. By performing $\frac{\varepsilon}{p}-$ Dehn surgery along $\partial D^{2}$, we have a new knot. The new knot is said to be the knot obtained from $K_{0}$ by an ( $\left.\varepsilon p, l\right)$-twisting. Let $\mathscr{K}_{p}$ be the set of knots obtained from a trivial knot by an ( $\varepsilon p, l)$-twisting for some integer $l$ and $\varepsilon= \pm 1$. Section 4 is devoted to two applications. Our first application is to find infinitely many knots that give a negative answer to the following question given by Mathieu [12]. $\mathscr{S}_{p}$ ?

Question 2. For any knot $K$, is there a positive integer $p$ such that $K \in$

Our second one is to find infinitely many counterexamples to the following conjecture made by Akbulut and Kirby.

Conjecture. If $K$ is a knot with Arf invariant zero, then $K$ is obtained from a slice knot by a $( \pm 1, \pm 1)$-twisting. (Problem 1.46 (B) of [9].)

It is shown that a $(2,7)$-torus knot cannot be obtained from a ribbon knot by a $(||$,$) -twisting by using Donaldson's outstanding theorem [ 1$, Theorem 1$]$ (see [10]). Since then Donaldson improved this result to drop "simply connectedness assumption" [2, Theorem 1], a $(2,7)$-torus knot cannot be obtained from a slice knot by a $(||$,$) -twisting. Here we give infinitely many$ counterexamples in different knot cobordism classes.

Similar results for Question 2 were obtained independently by Katura Miyazaki[13].

\section{PRELIMINARIES}

In this section we introduce some useful lemmas to us. In particular, Lemmas 1.8 and 1.11 are key lemmas in this paper. 
Let $\alpha, \beta$ be the standard generators of $H_{2}\left(S^{2} \times S^{2}\right)$ with $\alpha^{2}=\beta^{2}=0$, $\alpha \cdot \beta=1$ and let $\gamma$ or $\gamma_{i}$ (resp. $\bar{\gamma}$ or $\left.\bar{\gamma}_{i}\right)$ be the standard generator of $H_{2}\left(C P^{2}\right)$ (resp. $H_{2}\left(\overline{C P^{2}}\right)$ ) with $\gamma^{2}=\gamma_{i}^{2}=\mathrm{I}\left(\right.$ resp. $\bar{\gamma}^{2}=\bar{\gamma}_{i}^{2}=-1$ ). From now on a homology class in $H_{2}\left(M-\right.$ Int $\left.B^{4}, \partial\right)$ is identified with its image by the homomorphism

$$
H_{2}\left(M-\operatorname{Int} B^{4}, \partial\right) \cong H_{2}\left(M-\operatorname{Int} B^{4}\right) \rightarrow H_{2}(M) .
$$

Let $l$ and $m$ be nonnegative integers and $\varepsilon= \pm 1$. An $(\varepsilon l, m)$-lorus link is the link that wraps around the standardly embedded solid torus in $S^{3}$ in the longitudinal direction $l$ times and in the meridional direction $m$ times, where the intersection number of the meridian and longitude is $\varepsilon$. When $l$ and $m$ are relatively prime, it is a knot and called an $(\varepsilon l, m)$-torus knot. An $(\varepsilon l, m)$-torus knot is denoted by $T(\varepsilon l, m)$.

Let $L$ be a $\mu$-component link in $S^{3}$. Let $f_{i}: I \times I \rightarrow S^{3}, i=1, \ldots, m-1(m \leq \mu)$ be mutually disjoint embeddings such that

(i) $f_{i}(I \times I) \cap L=f_{i}(I \times \partial I)$ for each $i(i=1, \ldots, m-1)$ and

(ii) the link $L^{\prime}=C l\left(L \cup \cup f_{i}(\partial I \times I)-\cup f_{i}(I \times \partial I)\right)$ has the orientation compatible with that of $L-\cup f_{i}(I \times \partial I)$ and $\cup f_{i}(\partial I \times I)$.

The link $L^{\prime}$ is said to be the link obtained from $L$ by $m$-fusion if the number of the components of $L^{\prime}$ is $\mu-m$. In particular if the number of the components of $L^{\prime}$ is one, then $L^{\prime}$ is said to be the knot obtained from $L$ by complete fusion. We call the images $f_{1}(I \times I), \ldots, f_{m}(I \times I)$ the strips connecting $L$. Let $\mathscr{T}_{\varepsilon, x}(\varepsilon= \pm 1, x \geq 0)$ be the set of knots obtained from a $(2 \varepsilon, 4 x)$-torus link by 1 -fusion. Note that a knot $K$ belongs to $\mathscr{T}_{x}$ if and only if the reflected inverse $-K^{!}$belongs to $\mathscr{T}_{-x}$.

1.1. Lemma. For any knor $K \in \mathscr{T}_{x x}$, there exists an embedded 2-disk $\Delta$ in $S^{2} \times S^{2}-$ Int $B^{4}$ such that $\Delta$ represents an element $2 \alpha+2 \varepsilon x \beta$ in $H_{2}\left(S^{2} \times S^{2}-\right.$ Int. $\left.B^{4}, \partial\right)$ and $\partial \Delta \subset \partial\left(S^{2} \times S^{2}-\right.$ Int. $\left.B^{4}\right)$ is $-K^{!}$.

Proof. We first deal with the case that $K \in \mathscr{T}_{x}$. It is easily seen that there exist mutually disjoint $2 x+2$ properly embedded 2-disks $\Delta_{1}, \ldots, \Delta_{2 x+2}$ in $S^{2} \times S^{2}-$ Int $B^{4}$ such that $\cup \Delta_{i}$ represents an element $2 \alpha+2 x \beta$ and $\partial\left(\cup \Delta_{i}\right) \subset$ $\partial\left(S^{2} \times S^{2}-\right.$ Int $\left.B^{4}\right)$ is a Figure 1. Since a $(-2,4 x)$-torus link is obtained from $\partial\left(\cup \Delta_{i}\right)$ by $2 x$-fusion, there exist $2 x+1$ strips $b_{1}, \ldots, b_{2 x+1}$ connecting the link $\partial\left(\cup \Delta_{i}\right)$ such that $\Delta=\Delta_{1} \cup \ldots \cup \Delta_{2 x+2} \cup b_{1} \cup \ldots \cup b_{2 x+1}$ is an embedded 2-disk in $S^{2} \times S^{2}-$ Int $B^{4}$ and $\partial \Delta \subset\left(S^{2} \times S^{2}-\operatorname{Int} B^{4}\right)$ is $-K$ !

The above argument remains valid in case $K \in \mathscr{F}_{-x}$ 


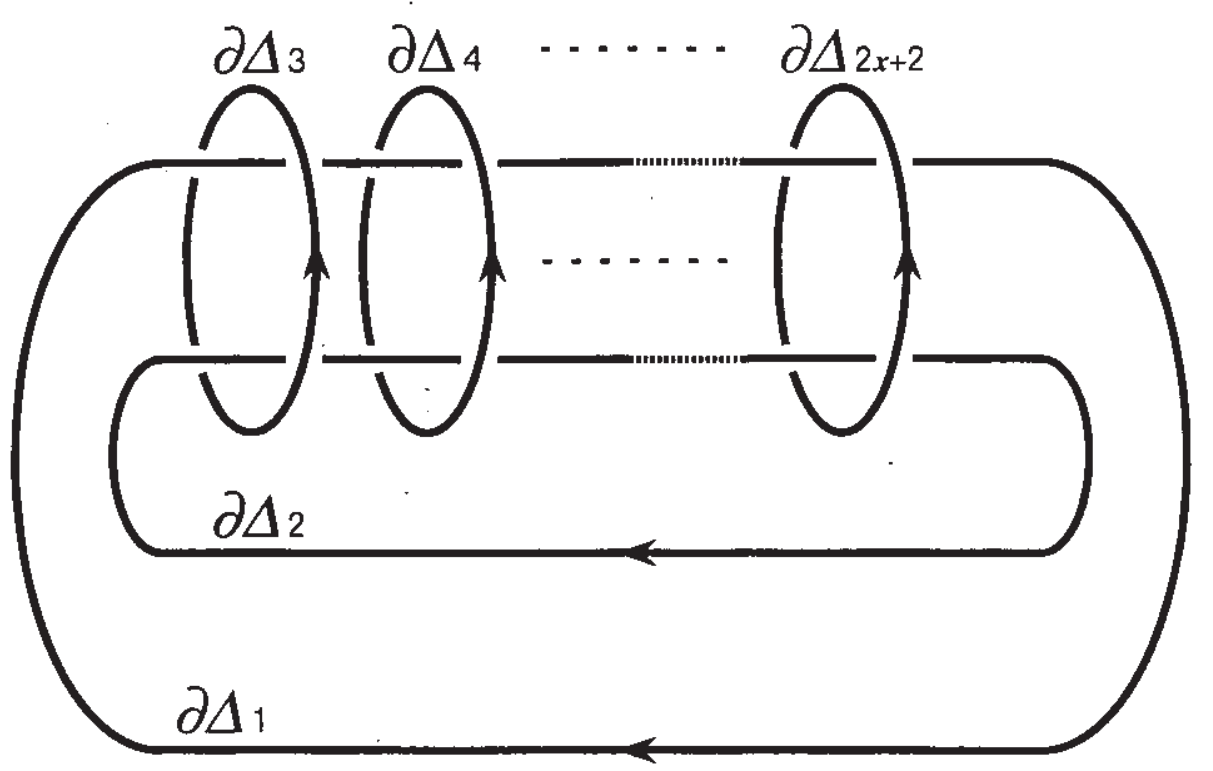

Figure 1

1.2. Lemma. For any knot $K \in \mathscr{T}_{\varepsilon x}$, there exists an embedded 2-disk $\Delta$ in $C P^{2} \# \overline{C P^{2}}-$ Int $B^{4}$ such that $\Delta$ represents an element $(2 x+\varepsilon) \gamma+(2 x-\varepsilon) \bar{\gamma}$ in $\mathrm{H}_{2}\left(C P^{2} \# \overline{C P^{2}}-\right.$ Int. $\left.B^{4}, \partial\right)$ and $\partial \Delta \subset \partial\left(C P^{2} \# \overline{C P^{2}}-\operatorname{lnt} B^{4}\right)$ is $-K^{\mathrm{I}}$ :

Proof. We first deal with the case that $K \in \mathscr{T}_{x}$. Let $O_{j} \cup O_{-1}$ be a 2-component trivial link in $\partial B^{4}$ such that $O_{j}$ is framed by $j(j= \pm 1)$. By considering the "Kirby's calculus"[8] as Figure 2, we note that there exist mutually disjoint $2 x+1$ properly embedded 2-disks $\Delta_{1}, \ldots, \Delta_{2 x+1}$ in $C P^{2} \# \overline{C P^{2}}-$ Int $B^{4}$ such that $\cup \Delta_{i}$ represents an element $(2 x+1) \gamma+(2 x-1) \bar{\gamma}$ in $H_{2}\left(C P^{2} \# \overline{C P^{2}}-\right.$ Int $\left.B^{4}, \partial\right)$ and $\partial\left(\cup \Delta_{i}\right) C \partial\left(C P^{2} \# \overline{C P^{2}}-\right.$ Int $\left.B^{4}\right)$ is as Figure 3. Since a $(-2,4 x)$-torus link is obtained from $\partial\left(\cup \Delta_{i}\right)$ by $(2 x-1)$-fusion, there exist $2 x$ strips $b_{1}, \ldots, b_{2 x}$ connecting the link $\partial\left(U \Delta_{i}\right)$ such that $\Delta=\Delta_{1} \cup \ldots \cup \Delta_{2 x+1} \cup b_{1} \cup \ldots \cup b_{2 x}$ is an embedded 2-disk in $C P^{2} \# \overline{C P^{2}}-\operatorname{Int} B^{4}$ and $\partial \Delta C \partial\left(C P^{2} \# \overline{C P^{2}}-\right.$ Int $\left.B^{4}\right)$ is $-K^{!}$.

By considering the Kirby's calculus as in Figure 4, the above argument remains valid in case $K \in \mathscr{T}_{-x}$

1.3. Lemma. (Rohlin [16]) Let $M$ be a connected, simply connected, closed 4-manifold. If $\xi \in H_{2}(M)$ is represented by an embedded 2-sphere in $M$, then 


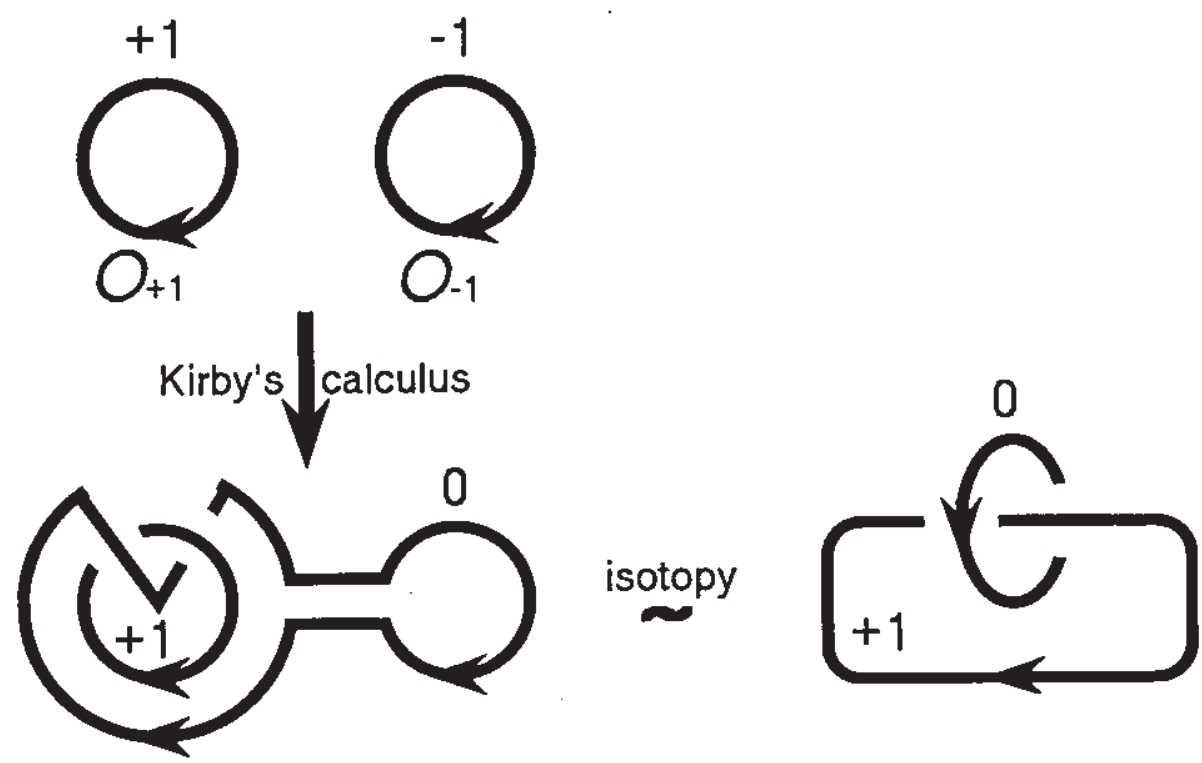

Figure 2

(a) $\left|\frac{\xi^{2}}{2}-\sigma(M)\right| \leq \operatorname{rank} H_{2}(M)$ if $\xi$ is divisible by 2 ,

(b) $\left|\frac{\xi^{2}\left(q^{2}-1\right)}{2 q^{2}}-\sigma(M)\right| \leq \operatorname{rank} H_{2}(M)$ if $\xi$ is divisible by an odd prime

integer $q$, where $\sigma(M)$ is the signature of $M$.

1.4. Lemma. (Weintraub [18], Yamamoto [19]) Let $K$ be a knot. If the unknotting number of $K$ is less than or equal to $u$ then there exists embedded 2-disk $\Delta$ in $u\left(C P^{2} \# C P^{2}\right)$ - Int $B^{4}$ such that $\Delta$ represents the zero element in $H_{2}\left(u\left(C P^{2} \sharp \overline{C P^{2}}\right)-\right.$ Int $\left.B^{4}, \partial\right)$ and $\partial \Delta C \partial\left(u\left(C P^{2} \# \overline{C P^{2}}\right)-\right.$ Int $\left.B^{4}\right)$ is $-K^{!}$.

1.5. Lemma. (Lawson [11]) Let $\xi \in H_{2}\left(C P^{2} \# 2 \overline{C P^{2}}\right)$ be a characteristic element. The element $\xi$ is represented by a 2 -sphere in $C P^{2} \# 2 \overline{C P^{2}}$ if and only if $\xi^{2}=-1$.

1.6. Lemma. (Lawson [11]) Let $\xi \in H_{2}\left(C P^{2} \# n \overline{C P^{2}}\right)(n \geq 3)$ be a characteristic element. If $\xi$ is represented by a 2-sphere in $C P^{2} \# n \overline{C P^{2}}$ then $\xi^{2} \leq-2$. 


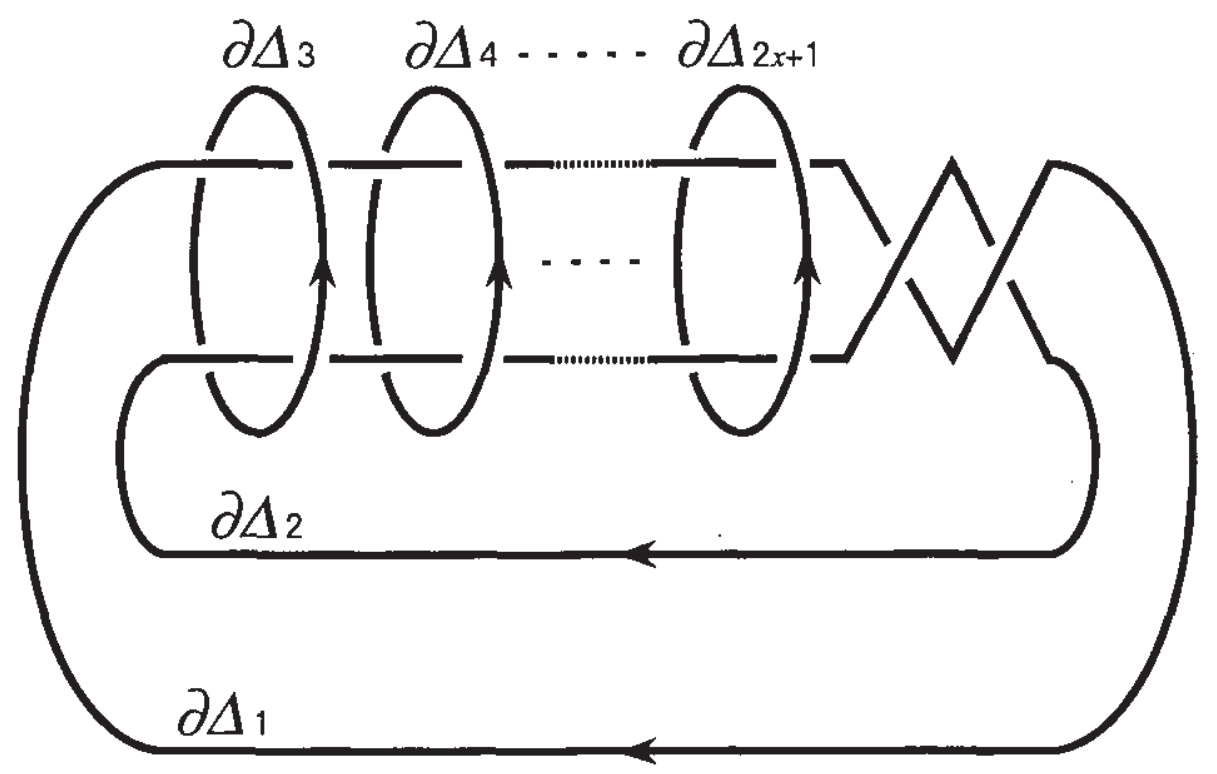

Figure 3
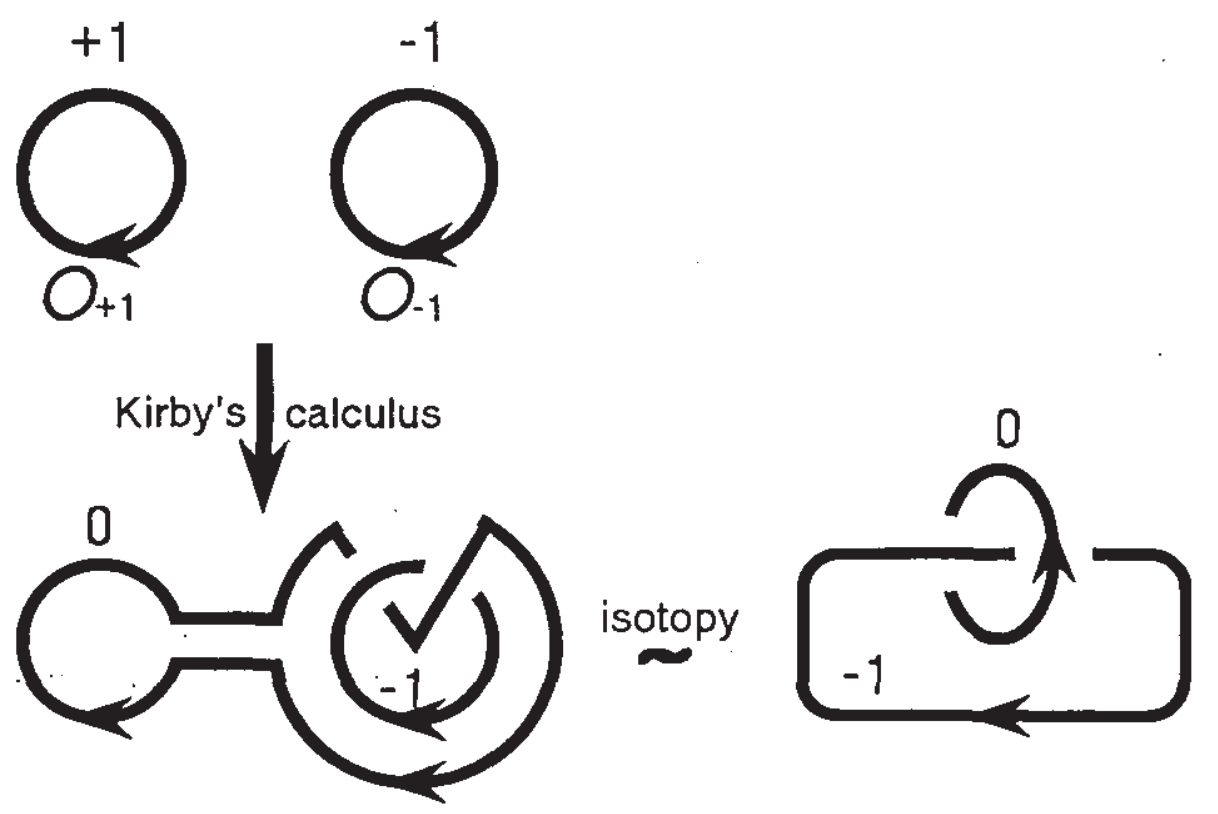

Figure 4 
1.7. Lemma. (Kikuchi [7]) Let $\xi \in H_{2}\left(C P^{2} \# 3 \overline{C P^{2}}\right)$ be a characteristic element. The element $\xi$ is represented by a 2 -sphere in $C P^{2} \# 3 C P^{2}$ if and only if $\xi^{2}=-2$.

1.8. Lemma. Let $p$ be a positive integer and $x$ a nonnegative integer. Let $K \in \mathscr{T}_{x}$ be a knot such that the unknotting number of $K$ is less than or equal to $u$. If $K \in e-$ Slice $\left(p \overline{C P^{2}}\right)$ then there exists an integer $z$ such that $z$ satisfies a condition

(a) $\frac{8 x-4}{p} \leq z^{2} \leq \frac{4 u}{p}+4$ and $z$ is even, or

(b)

$$
\left\{\begin{array}{l}
z^{2}=8 x+1 \text { if } p=1 \\
z^{2}=4 x+1 \text { if } p=2 \\
\frac{8 x+z}{p} \leq z^{2} \leq \frac{9}{2}\left(\frac{u}{p}+1\right) \text { and } z \text { is odd if } p \geq 3 .
\end{array}\right.
$$

Proof. Suppose that $K \in \mathscr{F}_{x} \cap e$-Slice $\left(p \overline{C P^{2}}\right)$ and the unknotting number of $K$ is less than or equal to $u$. Since $K \in \mathscr{F}_{x} \cap e-$ Slice $\left(p \overline{C P^{2}}\right)$, there exists an integer $z$ such that

(1) $2 \alpha+2 x \beta+z\left(\bar{\varepsilon}_{1} \bar{\gamma}_{1}+\ldots+\bar{\varepsilon}_{p} \bar{\gamma}_{p}\right) \in H_{2}\left(S^{2} \times S^{2} \# p \overline{C P^{2}}\right)$ is represented by a 2-sphere in $S^{2} \times S^{2} \# p \overline{C P^{2}}$ and

(2) $(2 x+1) \gamma+(2 x-1) \bar{\gamma}+z\left(\bar{\varepsilon}_{1} \bar{\gamma}_{1}+\ldots+\bar{\varepsilon}_{p} \bar{\gamma}_{p}\right) \in H_{2}\left(C P^{2} \#(p+1) \overline{C P^{2}}\right)$ is represented by a 2 -sphere in $C P^{2} \#(p+1) \overline{C P^{2}}$,

by Lemmas 1.1, 1.2 and the definition of evenly slice knots. Since the unknotting number of $K$ is less than or equal to $u$, by Lemma 1.4,

(3) $z\left(\bar{\varepsilon}_{1} \bar{\gamma}_{1}+\ldots+\bar{\varepsilon}_{p} \bar{\gamma}_{p}\right)$ is represented by a 2 -sphere in $p \overline{C P^{2}} \# u\left(C P^{2} \# \overline{C P^{2}}\right)$.

In case that $z$ is even. By Lemma 1.3, (1) and (3),

$$
\begin{gathered}
\left|\frac{8 x-p z^{2}}{2}+p\right| \leq p+2, \\
\left|\frac{-p z^{2}}{2}+p\right| \leq p+2 u
\end{gathered}
$$


It follows that

$$
\frac{8 x-4}{p} \leq z^{2} \leq \frac{4 u}{p}+4 .
$$

In case that $z$ is odd and $|z| \geq 3$. By Lemma 1.3 and (3), there exists an odd prime integer $q$ such that

$$
\left|\frac{-p z^{2}\left(q^{2}-1\right)}{2 q^{2}}+p\right| \leq p+2 u .
$$

This implies

$$
z^{2} \leq \frac{9}{2}\left(\frac{u}{p}+1\right)
$$

We note that

$$
1<\frac{9}{2}\left(\frac{u}{p}+1\right) .
$$

The inequations (1-1) and (1-2) imply that any odd integer $z$ satisfies

$$
1 \leq z^{2} \leq \frac{9}{2}\left(\frac{u}{p}+1\right) .
$$

Moreover if $z$ is odd then $(2 x+1) \gamma+(2 x-1) \bar{\gamma}+z\left(\bar{\varepsilon} \bar{\gamma}_{1}+\ldots+\bar{\varepsilon}_{p} \bar{\gamma}_{p}\right)$ is a characteristic element in $H_{2}\left(C P^{2} \#(p+1) \overline{C P^{2}}\right)$. By Lemmas $1.5,1.6,1.7$ and (2),

$$
8 x-z^{2}=-1 \text { if } p=1 \text {, }
$$

$$
8 x-2 z^{2}=-2 \text { if } p=2,
$$

$$
8 x-p z^{2} \leq-2 \text { if } p \geq 3 \text {. }
$$

By (1-3), (1-4), (1-5) and (1-6), we have

$$
\begin{aligned}
& z^{2}=8 x+1 \text { if } p=1, \\
& z^{2}=4 x+1 \text { if } p=2,
\end{aligned}
$$




$$
\frac{8 x+2}{p} \leq z^{2} \leq \frac{9}{2}\left(\frac{u}{p}+1\right) \text { if } p \geq 3 .
$$

This completes the proof.

Suppose that knots $K_{+}$and $K_{-}$have representatives in $S^{3}$ that are identical outside a 3-ball within which they are as in Figure 5. Then we say that $K_{-}$is obtained from $K_{+}$by changing a positive crossing and that $K_{+}$is obtained from $K_{-}$by changing a negative crossing. We define the positive unknotting number (resp. negative unknotting number) of a knot $K$, to be the minimum, over all sequences transforming $K$ to be a trivial knot, of the number of positive (resp. negative) crossings which are changed. If $K$ cannot be a trivial knot by changing only positive (resp. negative) crossings, then we define the positive unknotting number (resp. negative unknotting number) of $K$ is infinite.
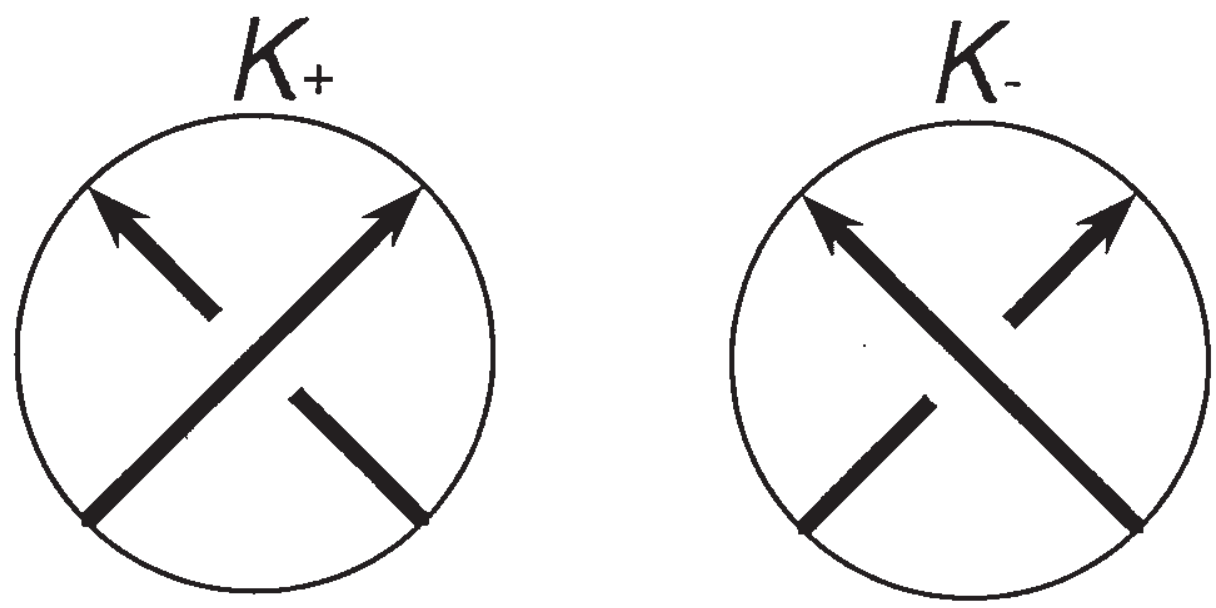

Figure 5

1.9. Lemma. (Weintraub [18]) Let $K$ be a knot. If the positive unknotting number (resp. negative unknotting number) of $K$ is less than or equal to $u$, then there exists an embedded 2-disk $\triangle$ in $u \overline{C P^{2}}-\operatorname{Int} B^{4}$ (resp. $\left.u C P^{2}-\operatorname{Int} B^{4}\right)$ such that $\triangle$ represents the zero element in $H_{2}\left(u \overline{C P^{2}}-\right.$ Int $\left.B^{4}, \partial\right)$ (resp. $H_{2}\left(u C P^{2}-\right.$ Int $\left.\left.B^{4}, \partial\right)\right)$ and $\partial \Delta \subset \partial\left(u \overline{C P^{2}}-\right.$ Int $\left.B^{4}\right)\left(\right.$ resp. $\left.\partial \Delta \subset \partial\left(u C P^{2}-\operatorname{lnt} B^{4}\right)\right)$ is $-K$ !

1.10. Lemma. (Kervaire and Milnor [6]) Let $M$ be a connected, simply connected, closed 4-manifold. Let $\xi \in H_{2}(M)$ be a characteristic element. If $\xi$ is represented by an embedded 2-sphere in $M$, then $\xi^{2} \equiv \sigma(M) \bmod 16$. 
1.11. Lemma. Let $p$ be a positive integer and $x$ a nonnegative integer. Let $K \in \mathscr{T}_{-x}$ be a knot such that the negative unknotting number of $K$ is finite. If $K \in e-$ Slice $\left(p \overline{C P^{2}}\right)$ then there exists an integer $z$ such that $z$ satisfies a condition

(a) $z^{2} \leq 4+\frac{4-8 x}{p}$ and $z$ is even, or

(b) $\left\{\begin{array}{l}z^{2}=1 \text { only if } x=0 \text { and } p=1,2, \\ z^{2}=1 \text { only if } x \equiv 0 \bmod 2 \text { and } p \geq 3 .\end{array}\right.$

Proof. Suppose $K \in \mathscr{T}_{-x} \cap e$-Slice $\left(p \overline{C P^{2}}\right)$ and the negative unknotting number of $K$ is $u$. Since $K \in \mathscr{T}_{-x} \cap e$-Slice $\left(p \overline{C P^{2}}\right)$, there exists an integer $z$ such that

(4) $2 \alpha-2 x \beta+z\left(\bar{\varepsilon}_{1} \bar{\gamma}_{1}+\ldots \pm \bar{\varepsilon}_{p} \bar{\gamma}_{\mu}\right) \in H_{2}\left(S^{2} \times S^{2} \# p \overline{C P^{2}}\right)$ is represented by a 2-sphere in $S^{2} \times S^{2} \# p \overline{C P^{2}}$ and

(5) $(2 x-1) \gamma+(2 x+1) \bar{\gamma}+z\left(\bar{\varepsilon}_{1} \bar{\gamma}_{1}+\ldots+\bar{\varepsilon}_{p} \bar{\gamma}_{p}\right) \in H_{2}\left(C P^{2} \#(p+1) \overline{C P^{2}}\right)$ is represented by a 2 -sphere in $C P^{2} \#(p+1) \overline{C P^{2}}$,

by Lemmas $1.1,1.2$ and the definition of evenly slice knots. Since the negative unknotting number of $K$ is $u$, by Lemma 1.9 ,

(6) $z\left(\bar{\varepsilon}_{1} \bar{\gamma}_{1}+\ldots+\bar{\varepsilon}_{p} \bar{\gamma}_{p}\right)$ is represented by a 2 -sphere in $p \overline{C P^{2}}$ \# $u C P^{2}$.

In case that $z$ is even. By Lemma 1.3 and (4),

$$
\left|-\frac{-8 x-p z^{2}-}{2}+p\right| \leq p+2 \text {. }
$$

This implies

$$
z^{2} \leq 4+\frac{4-8 x}{p}
$$

In case that $z$ is odd. If $|z| \geq 3$, then by Lemma 1,3 and (6), there exists an odd prime integer $q$ such that

$$
\left|\frac{-p z^{2}\left(q^{2}-1\right)}{2 q^{2}}+p-u\right| \leq p+u
$$


It follows that

$$
z^{2} \leq \frac{9}{2}
$$

This is a contradiction. Thus $|z|=1$. Moreover, by Lemmas $1.5,1.7,1.10$ and (5), we have

$$
\begin{aligned}
& -8 x-p z^{2}=-p \text { if } p=1,2 \\
& -8 x-p z^{2} \equiv-p \bmod 16
\end{aligned}
$$

Since $|z|=1$,

$$
\begin{aligned}
& -8 x=0 \text { if } p=1,2, \\
& -8 x \equiv \equiv 0 \bmod 16 .
\end{aligned}
$$

This implies

$$
\begin{gathered}
x=0 \text { if } p=1,2, \\
x \equiv 0 \bmod 2 .
\end{gathered}
$$

This completes the proof.

\section{SLICE KNOTS IN $C P^{2}$ or $\overline{C P^{2}}$}

In this section we shall prove the following two theorems.

2.1. Theorem. Let $x$ be a positive integer.

(a) If Slice $\left(\overline{C P^{2}}\right)$ contains $T(2,4 x-1)$, then $2 x-1,2 x$ or $8 x+1$ is a square number.

(b) If Slice $\left(\overline{C P^{2}}\right)$ contains $T(2,4 x+1)$, then $2 x, 2 x+1$ or $8 x+1$ is a square number.

2.2. Theorem. Let $t$ be a nonnegative integer. The set Slice $\left(\overrightarrow{C P^{2}}\right)$ does not contain $T(-2,2 t+1)$ if and only if $t \geq 2$.

2.3. Remark. Since Slice $\left(C P^{2}\right)$ contains a knot $K$ if and only if Slice $\left.\overline{\left(C P^{2}\right.}\right)$ contains $-K^{!}$, Slice $\left(C P^{2}\right)$ contains $T(l, m)$ if and only if 
Slice $\left(\overline{C P^{2}}\right)$ contains $T(-l, m)$. It follows that Theorems 2.1 and 2.2 imply that there exist infinitely many integer $x_{i}(i=1,2, \ldots)$ such that $T\left(2,2 x_{i}+1\right)$ belongs to neither Slice $\left(C P^{2}\right)$ nor Slice $\left(C P^{2}\right)$ for any $x_{i}$.

2.4. Lemma. For any $T(2 \varepsilon, 4 x+1)(\varepsilon= \pm 1, x \geq 0)$, there exists an embedded 2-disk $\triangle$ in $C P^{2} \# \overline{C P^{2}}-$ Int $B^{4}$ such that $\Delta$ represents an element $(2 x+1+\varepsilon) \gamma+(2 x+1-\varepsilon) \bar{\gamma}$ in $H_{2}\left(C P^{2} \# C P^{2}-\right.$ Int $\left.B^{4}, \partial\right)$ and $\partial \Delta C \partial\left(C P^{2} \# \overline{C P^{2}}-\right.$ Int $\left.B^{4}\right)$ is $T(-2 \varepsilon, 4 x+1)$.

Proof. By considering the Kirby's calculus as in Figure 2, we note that there exist mutually disjoint $2 x+2$ properly embedded 2 -disk $\Delta_{1}, \ldots, \Delta_{2 x+2}$ in $C P^{2} \# C P^{2}-$ Int $B^{4}$ such that $U \Delta_{i}$ represents an element $(2 x+2) \gamma+2 x \bar{\gamma}$ in $H_{2}\left(C P^{2} \# \overline{C P^{2}}-\right.$ Int $\left.B^{4}, \partial\right)$ and $\partial\left(\cup_{\Delta_{i}}\right) \subset \partial\left(C P^{2} \# \overline{C P^{2}}-\right.$ Int $\left.B^{4}\right)$ is as Figure 6. Since a $(-2,4 \dot{x}+2)$-torus link is obtained from $\partial\left(\cup_{\Delta_{i}}\right)$ by $2 x$-fusion, there exist $2 x+1$ strips $b_{1}, \ldots, b_{2 x+1}$ connecting the link $\partial\left(\cup_{\Delta_{i}}\right) \underline{\text { such that }} \Delta=\Delta_{1}$ $\cup \ldots \cup \Delta_{2 x+2} \cup b_{1} \cup \ldots \cup b_{2 x+1}$ is an embedded 2-disk in $C P^{2} \# C P^{2}-$ Int $B^{4}$ and $\partial \Delta C \partial\left(C P^{2} \# C P^{2}-\operatorname{lnt} B^{4}\right)$ is $T(-2,4 x+1)$.

By considering the Kirby's calculus as in Figure 4, the above argument remains valid for $T(-2,4 x+1)$.

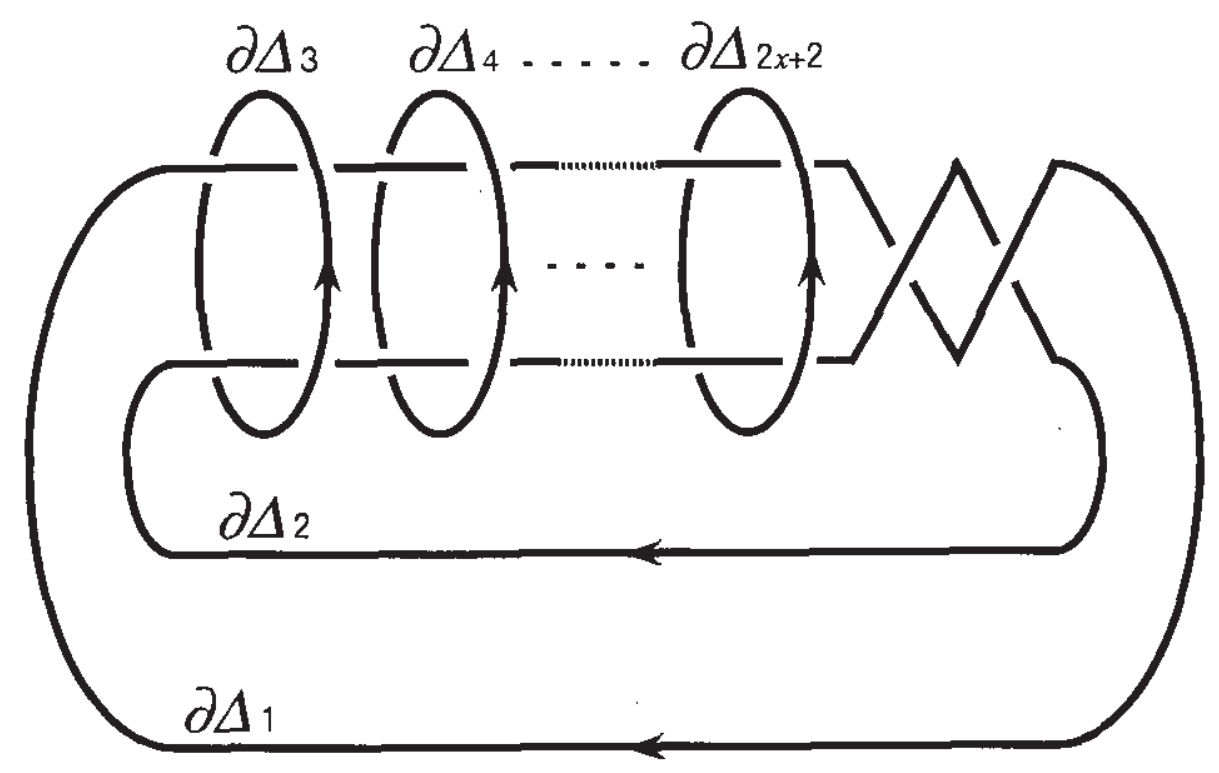

Figure 6 
Proof of Theorem 2.1. Suppose $T(2,4 x-1) \in$ Slice $\left(\overline{C P^{2}}\right)$. Since the unknotting number of $T(2,4 x-1)$ is $2 x-1, T(2,4 x-1) \in \mathscr{T}_{x}$ and e-Slice $\left(\overline{C P^{2}}\right)=$ Slice $\left(\overline{C P^{2}}\right)$, by Lemma 1.8 , there exists an integer $z$ such that $z$ satisfies a condition

$$
8 x-4 \leq z^{2} \leq 8 x \text { and } z \text { is even, or }
$$

$$
z^{2}=8 x+1
$$

We set $z=2 k$ in (2-7), then we have

$$
2 x-1 \leq k^{2} \leq 2 x \text {. }
$$

It follows that

$$
k^{2}=2 x-1,2 x \text {. }
$$

By (2-8) and (2-9), we obtain Theorem 2.1 (a).

Suppose $T(2,4 x+1) \in$ Slice $\left(\overline{C P^{2}}\right)$. Since the unknotting number of $T(2,4 x+1)$ is $2 x$ and $T(2,4 x+1) \in \mathscr{T}_{x}$, by L.Emma 1.8 , there exists an integer $z$ such that $z$ satisfies a condition

$$
8 x-4 \leq z^{2} \leq 8 x+4 \text { and } z \text { is even, or }
$$

$$
z^{2}=8 x+1 .
$$

The fact that $T(2,4 x+1)$ belongs to Slice $\left.\overline{\left(C P^{2}\right.}\right)$ and Lemma 2.4 imply that $(2 x+2) \gamma+2 x \bar{\gamma}+z \bar{\gamma}_{1} \in H_{2}\left(C P^{2} \# 2 \overline{C P^{2}}\right)$ is represented by a 2 -sphere in $C P^{2} \# 2 \overline{C P^{2}}$. If $z$ is even, then by Lemma 1.3, we have

$$
\left|\frac{8 x+4-z^{2}}{2}+1\right| \leq 3
$$

This implies

$$
8 x \leq z^{2} \leq 8 x+12
$$

By (2-10) and (2-12), we have

$$
8 x \leq z^{2} \leq 8 x+4 \text { and } z \text { is even. }
$$

We set $z=2 k$ in $(2-13)$ then

$$
2 x \leq k^{2} \leq 2 x+1
$$


It follows that

$$
k^{2}=2 x, 2 x+1
$$

By (2-11) and (2-14), we obtain Theorem 2.1 (b).

2.5. Proposition. If $t \geq 3$ then slice $\left(\overline{C P^{2}}\right)$ does not contain $T(-2$, $2 t+1)$.

Proof. Note that $\mathscr{T}_{-x}$ contains both $T(-2,4 x-1)$ and $T(-2,4 x+1)$ and that the negative unknotting number of $T(-2,4 x-1)$ and that the negative unknotting number of $T(-2,4 x+1)$ are finite. If Slice $\left(\overline{C P^{2}}\right)$ contains $T(-2,4 x-1)$ or $T(-2,4+1)$, then by Lemma 1.11 , there exists an integer $z$ such that $z$ satisfies a condition

$$
z^{2}=8-8 x \text { and } z \text { is even, or }
$$

$$
z^{2}=1 \text { and } x=0 \text {. }
$$

The conditions (2-15) and (2-16) imply

$$
x=0,1 \text {. }
$$

This completes the proof.

2.5.1. Remark. By the proofs of Lèmma 4.11 and Proposition 2.5, we note that if S/ice $\left(C P^{2}\right)$ contains $T(-2,5)$ then there exists a properly embedded 2-disk $\Delta$ in $\overline{C P^{2}}-\operatorname{Int} B^{4}$ such that $\Delta$ represents the zero element in $H_{2}\left(\overline{C P^{2}}-\operatorname{lnt} B^{4}, \partial\right)$ and $\partial \Delta C \partial\left(\overline{C P^{2}}-\operatorname{lnt} B^{4}\right)$ is $T(-2,5)$.

2.6. Proposition. The set Slice $\left(\overline{C P^{2}}\right)$ does not contain $T(-2,5)$.

Proof. Suppose Slice $\left(\overline{C P^{2}}\right)$ contains $T(-2,5)$. Remark 2.5 .1 and Lemma 2.4 imply that $2 \gamma+4 \bar{\gamma} \in H_{2}\left(C P^{2} \# \overline{C P^{2}}\right)$ is represented by a 2 -sphere in $C P^{2} \# 2 \overline{C P^{2}}$. By Lemma 1.3 , we have

$$
\left|\frac{4-16}{2}+1\right| \leq 3
$$

This is a contradiction. 
Proof of Theorem 2.2. By Propositions 2.5 and 2.6, if $t \geq 2$ then Slice $\overline{\left(\overline{C P^{2}}\right)}$ does not contain $T(-2,2 t+1)$. If $t=0$ or 1 then Slice $\left(\overline{C P^{2}}\right)$ contains $T(-2,2 t+1)$, see Proposition 3.7.

\section{EVENLY SLICE KNOTS IN $\mathrm{n}_{1} \mathrm{CP}^{2} \# \mathrm{n}_{2} \overline{\mathrm{CP}^{2}}$}

In [15], Norman proved that Slice $\left(C P^{2} \sharp \overline{C P^{2}}\right)$ is equal to the set of knots, but the following theorem implies that there exist infinitely many knots that do not belong to e-Slice $\left(C P^{2} \# \overline{C P^{2}}\right)$, i.e., e-Slice $\left(C P^{2} \# \overline{C P^{2}}\right)$ is a proper subset of Slice $\left(C P^{2} \# \overline{C P^{2}}\right)$.

3.1. Theorem, Let $t$ be a nonnegative integer and $\varepsilon= \pm 1$. The set e-Slice $\left(C P^{2} \# \overline{C P^{2}}\right)$ contains $T(2 \varepsilon, 2 t+1)$ if and only if $t=0$ or 1 .

3.2. Lemma. (Hirai [4]) Let $\xi \in H_{2}\left(2\left(C P^{2} \# \overline{C P^{2}}\right)\right)$ be a characteristic element. The element $\xi$ represented by a 2 -sphere in $2\left(C P^{2} \#\left(\overline{C P^{2}}\right)\right.$ if and only if $\xi^{2}=0$.

3.3. Proposition. For $\varepsilon= \pm 1$, if $t \geq 3$ then e-Slice $\left(C P^{2} \# \overline{C P^{2}}\right)$ does not contain $T(2 \mathrm{\varepsilon}, 2 t+1)$.

Proof. Let $x$ be a nonnegative integer. If either $T(2 \varepsilon, 4 x-1)$ or $T(2 \varepsilon$, $4 x+1)$ belongs to $e$-Slice $\left(C P^{2} \# \overline{C P^{2}}\right)$ then there exists an integer $z$ such that

(7) $2 \alpha+2 \varepsilon x \beta+z\left(\varepsilon_{1} \gamma_{1}+\bar{\varepsilon}_{1} \bar{\gamma}_{1}\right) \in H_{2}\left(S^{2} \times S^{2} \# C P^{2} \# \overline{C P^{2}}\right)$ is represented by a 2-sphere in $S^{2} \times S^{2} \# C P^{2} \# \overline{C P^{2}}$ and

(8) $(2 x+\varepsilon) \gamma+(2 x-\varepsilon) \bar{\gamma}+z\left(\varepsilon_{1} \gamma_{\perp}+\tilde{\varepsilon}_{1} \bar{\gamma}_{1}\right) \in H_{2}\left(2\left(C P^{2} \# \overline{C P^{2}}\right)\right)$ is represented by a 2 -sphere in $2\left(C P^{2} \# C P^{2}\right)$,

by Lemmas $1.1,1.2$ and the definition of evenly slice knots. If $z$ is even, then by Lemma 1.3 and (7),

$$
\left|\frac{8 \varepsilon x}{2}\right| \leq 4
$$

This implies

$$
x=0,1 \text {. }
$$


If $z$ is odd, then by Lemma 3.2 and ( 8 ),

$$
8 \varepsilon x=0 .
$$

It follows that if $x \geq 2$, then neither $T(2 \varepsilon, 4 x-1)$ nor $T(2 \varepsilon, 4 x+1)$ belongs to e-Slice $\left(C P^{2} \# \bar{C} P^{\overline{2}}\right)$. This completes the proof.

3.4. Proposition. The set e-Slice $\left(C P^{2} \# \overline{C P^{2}}\right)$ does not contain $T(2 \varepsilon, 5)$ for $\varepsilon= \pm 1$.

Proof. Suppose e-Slice $\left(C P^{2} \# \overline{C P^{2}}\right)$ contains $T(2 \varepsilon, 5)$. Proof of Proposition 3.3 and Lemma 2.4 implies that there exists an even integer $z$ such that $(3+\varepsilon) \gamma+(3-\varepsilon) \bar{\gamma}+z\left(\varepsilon_{1} \gamma_{1}+\bar{\varepsilon}_{1} \bar{\gamma}_{1}\right) \in H_{2}\left(2\left(C P^{2} \# \overline{C P^{2}}\right)\right)$ is represented by a $2-$ sphere in $2\left(C P^{2} \# \overline{C P^{2}}\right)$. By Lemma 1.3, we have

$$
\left|\frac{12 \varepsilon}{2}\right| \leq 4
$$

This is a contradiction.

Proof of Theorem 3.1. By Propositions 3.3 and 3.4, if $t \geq 2$ then $e$-Slice $\left(C P^{2} \# C P^{2}\right)$ does not contain $T(2 \varepsilon, 2 t+1)$. If $t=0$ or 1 then $e-$ Slice $\left(C P^{2} \# \overline{C P^{2}}\right)$ contains $T(2 \varepsilon, 2 t+1)$, see Proposition 3.7.

The same arguments as proof of Theorem 2.1 and Proposition 2.5 lead to the following Theorem 3.5 and Proposition 3.6, respectively.

3.5. Theorem. Let $x$ be a positive integer.

(a) If e-Slice $\left(2 \overline{C P^{2}}\right)$ contains $T(2,4 x-1)$ then $x$ or $4 x+1$ is a square number.

(b) If e-Slice $\left(2 \overline{C P^{2}}\right)$ contains $T(2,4 x+1)$ then $x, x+1$ or $4 x+1$ is a square number.

3.6. Proposition. If $t \geq 3$ then $e_{-}$Slice $\left(2 \overline{C P^{2}}\right)$ does not contain $T(-2$, $2 t+1)$

3.7. Proposition. Let $K$ be a knot. If the positive unknotting number or the negative unknoting number of $K$ is less than or equal to $p$, then both e-Slice $\left(p C P^{2}\right)$ and e-Slice $\left(p \overline{C P^{2}}\right)$ contain $K$. 
Proof. Suppose $K$ is a knot and the positive or negative unknotting number of $K$ is less than or equal to $p$. Let $L_{\mathrm{r}}$ be the Hopf link in $\partial\left(C P^{2}-\right.$ Int $\left.B^{4}\right)$ with linking number $\varepsilon(\varepsilon= \pm 1)$. It is easily seen that $L_{\mathrm{c}}$ bounds a properly embedded 2-disk in $C P^{2}-$ Int $B^{4}$ that represents an element $(\mathrm{l}-\varepsilon) \gamma$ in $\mathrm{H}_{2}\left(C P^{2}-\mathrm{Int} B^{4}, \partial\right)$. Since the positive or negative unknotting number of $K$ is less than or equal to $p, K$ is obtained from the $p$ copies of $L_{\mathrm{\varepsilon}}$ by complete fusion. It follows that $K$ bounds a properly embedded 2-disk in $p C P^{2}-\operatorname{Int} B^{4}$ that represents an element $(1-\varepsilon)$ $\left(\varepsilon_{1} \gamma_{1}+\ldots+\varepsilon_{p} \gamma_{p}\right)$ in $H_{2}\left(p C P^{2}-\right.$ Int $\left.B^{4}, \partial\right)$. This implies that $K$ belongs to $e$ Slice $\left(p C P^{2}\right)$.

The above argument remains valid to show that $K$ belongs to e-Slice $\left(p \frac{C P^{2}}{2}\right)$. This completes the proof.

By Propositions 3.6 and 3.7, we have the following theorem.

3.8. Theorem. Let $t$ be a nonnegative integer. The set e-Slice $\left(2 \overline{C P^{2}}\right)$ does not contain $T(-2,2 t+1)$ if and only if $t \geq 3$.

3.9. Theorem. For any integer $p \geq 3$, e-Slice $\left(p \overline{C P^{2}}\right)$ contains neither $T(2,8 p+3)$ nor $T(-2,8 p+3)$.

Proof. Suppose that $e$-Slice $\left(p \overline{C P^{2}}\right)$ contains $T(2,8 p+3)$. Since $T(2,8 p+3)$ belongs to $\mathscr{T}_{2 p+1}$ and the unknotting number of $T(2,8 p+3)$ is $4 p+1$, by Lemma 1.8 , there exists an integer $z$ such that $z$ satisfies a condition

$$
\begin{aligned}
& \frac{16 p+4}{p} \leq z^{2} \leq \frac{16 p+4}{p}+4 \text { and } z \text { is even, or } \\
& \frac{16 p+10}{p} \leq z^{2} \leq \frac{9}{2}\left(\frac{4 p+4}{p}+1\right) \text { and } z \text { is odd. }
\end{aligned}
$$

Since $p \geq 3,(3-17)$ and (3-18) imply

$$
\begin{aligned}
& 16<z^{2}<25 \text { and } z \text { is even, } \\
& 16<z^{2}<25 \text { and } z \text { is odd. }
\end{aligned}
$$

This is a contradiction.

Suppose that $e-$ Slice $\left(p \overline{C P^{2}}\right)$ contains $T(-2,8 p+3)$. Since $T(-2,8 p+3)$ belongs to $\mathscr{T}_{-2 p-1}$ and the negative unknotting number of $T(-2,8 p+3)$ is 
finite, by Lemma 1.11, there exists an integer $z$ such that $z$ satisfies the following condition

$$
z^{2} \leq 4+\frac{-16 p-4}{p-}<0
$$

This is a contradiction.

3.10. Claim. Let $K$ be a knot. Neither $e$-Slice $\left(p C P^{2}\right)$ nor $e$-Slice $\left(p \overline{C P^{2}}\right)$ contains $K$ if and only if $e$-Slice $\left(p \overline{C P^{2}}\right)$ contains neither $K$ nor $-K^{\prime}$.

3.11. Remark. By Theorem 3.9 and Claim 3.10, we have that $T(2,8 p+3)$ belongs to neither e-Slice $\left(p C P^{2}\right)$ nor e-Slice $\left(p \overline{C P^{2}}\right)$ for any $p \geq 3$.

\section{APPLICATIONS}

4.1. Proposition. If $K \in \mathscr{K}_{p}$ then $K$ belongs to either $e$-Slice $\left(p C P^{2}\right)$ or e-Slice $\left(p \overline{C P^{2}}\right)$.

Proof. If $K \in \mathscr{K}_{p}$ then there exists a 2-disk $D^{2}$ and a trivial knot $K_{0}$ in $S^{3}$ such that $K$ is obtained from $K_{0}$ by $\frac{\varepsilon}{p}$-Dehn surgery along $\partial D^{2}$. We take the parallel copies $D_{1}^{2}, \ldots, D_{p}^{2}$ of $D^{2}$ as in Figure 7. It is easily seen that $K$ is obtained from $K^{0}$ by Dehn.surgery along $\partial\left(\cup D_{i}^{2}\right)$ in which the surgery coefficients are all $\varepsilon$. Suppose that $K_{0}$ and $U D_{i}^{2}$ are in the boundary of a 4ball $B_{0}^{4}$, then $K_{0}$ bounds a properly embedded 2-disk $\Delta$ in $B_{0}^{4}$. Let $\left\{h_{i}^{2}\right\}$ $(1 \leq i \leq p)$ be 2 -handles on $B_{0}^{4}$ whose attaching sphere are $\left\{\partial D_{i}^{2}\right\}$ and all framings are $\varepsilon$. We note that $K_{0} \subset \partial\left(B_{0}^{4} \cup \cup h_{i}^{2}\right)$ is $K, K$ bounds the 2-disk $\Delta$ in $B_{0}^{4} \cup \cup h_{i}^{2}$ and $B_{0}^{4} \cup \cup h_{i}^{2}$ is deffeomorphic to either punctured $p C P^{2}$ or punctured $p \overline{C P^{2}}$. Let the punctured $p C P^{2}$ and punctured $p \overline{C P^{2}}$ be denoted by $p C P^{2}-$ Int $B^{4}$ and $p C P^{2}-$ Int $B^{4}$, respectively. Suppose the linking number $l k\left(K_{0}, \partial D^{2}\right)=z$ then $l k\left(K_{0}, \partial D_{i}^{2}\right)(1 \leq i \leq p)$ are the same number as $z$. It is not hard to see that $\Delta$ represents either an element $z\left(\varepsilon_{1} \gamma_{1}+\ldots+\varepsilon_{p} \gamma_{p}\right)$ in $H_{2}\left(p C P^{2}-\right.$ Int $\left.B^{4}, \partial\right)$ or an element $z\left(\bar{\varepsilon}_{1} \bar{\gamma}_{1}+\ldots \bar{\varepsilon}_{p} \bar{\gamma}_{p}\right)$ in $H_{2}\left(p \overline{C P^{2}}-\right.$ Int $\left.B^{4}, \partial\right)$. This implies that $K$ belongs to either e-Slice $\left(p C P^{2}\right)$ or $e$-Slice $\left(p \overline{C P^{2}}\right)$.

By Remark 3.11, Proposition 4.1 and the definition of evenly slice knots, we have the following theorem.

4.2. Theorem. For any integer $p \geq 3$, $\mathscr{K}_{p}$ does not contain any knot that is cobordant to $T(2,8 p+3)$. 

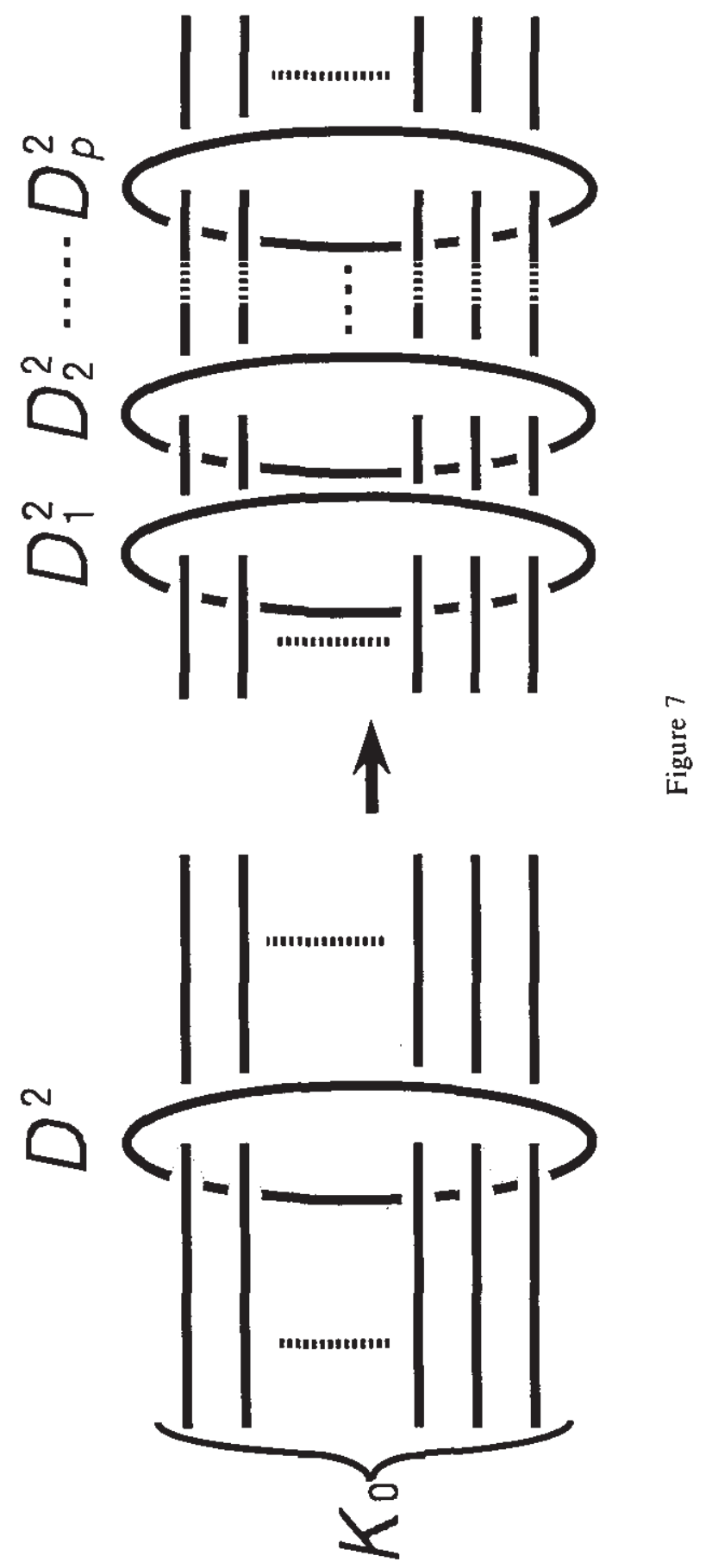
By Lemmas 1.8 and 1.11 , we have the following proposition.

4.3. Proposition. For any $p(1 \leq p \leq 5)$, e-Slice $\left(p \overline{C P^{2}}\right)$ contains neither $T(2,75)$ nor $T(-2,75)$.

By Claim 3.10, Propositions 4.1, 4.3 and the definition of evenly slice knots, we have the following proposition.

4.4. Proposition. For any $p(1 \leq p \leq 5)$, $\mathscr{K}_{p}$ does not contain any knot that is cobordant to $T(2,75)$.

4.5. Lemma. (Motegi [14]) If $p \geq 6$ then $\mathscr{K}_{p}$ does not contain any composite knot.

Let $K$ be a nontrivial slice knot. Proposition 4.4 and Lemma 4.5 imply that $\mathscr{H}_{p}$ does not contain $T(2,75) \# K$ for any $p \geq 1$. Hence we have the following theorem that gives a negative answer to Question 2.

4.6. Theorem. There exist infinitely many knots that do not belong to any $\mathscr{K}_{p}(p \geq 1)$.

Let $K$ be a knot in $\partial\left(C P^{2} \# \overline{C P^{2}}-\right.$ Int $\left.B^{4}\right)$. If $K$ is obtained from a slice knot by a ( $\pm \mathrm{I}, \pm \mathrm{l}$ )-twisting, then by proof of Proposition 4.1, $K$ bounds a properly embedded 2-disk in $C P^{2} \# \overline{C P^{2}}-$ Int $B^{4}$ that represents an element $\pm \gamma_{1}$ or $\pm \bar{\gamma}_{1}$ in $H_{2}\left(C P^{2} \# \overline{C P^{2}}-\operatorname{Int} B^{4}, \partial\right)$. It follows that $K$ bounds a properly embedded 2-disk in $C P^{2} \# \overline{C P^{2}}-$ Int $B^{4}$ that represent an element $\pm \gamma_{1}+\tilde{\gamma}_{1}$ or $\gamma_{1} \pm \bar{\gamma}_{1}$ in $\mathrm{H}_{2}\left(C P^{2} \# \overline{C P^{2}}-\right.$ Int $\left.B^{4}, \partial\right)$. We have the following proposition.

4.7. Proposition. If $K$ is obtained from a slice knot by $( \pm \mathrm{I}, \pm 1)$-twisting, then $K$ belongs to e-Slice $\left(C P^{2} \# \overline{C P^{2}}\right)$.

Since a $( \pm 1, \pm 1)$-twisting does not change the Arf invariant of a knot, thus $T(2 \varepsilon, 3)$ cannot be obtained from a slice knot by a ( $\pm 1, \pm 1)$-twisting. By Theorem 3.1, Proposition 4.7 and the definition of evenly slice knots, we have the following theorem.

4.8. Theorem. Let $t$ be a nonnegative integer and $\varepsilon= \pm 1$. A knot cobordant to $T(2 \mathrm{E}, 2 t+1)$ is obtained from a slice knot by $a( \pm 1, \pm 1)$-twisting if and only if $t=0$. 
If $2 t+1= \pm 1 \bmod 8$, then the Arf invariant of $T(2 \varepsilon, 2 t+1)$ is zero (for example, see p266 in [5]). Thus Theorem 4.8 gives infinitely many counterexamples to Conjecture.

Acknowledgement. The author would like to thank Professor Shin ichi Suzuki for his encouragement.

\section{References}

[1] S. K. DonaldSon: An application of gauge theory to four-dimensional topology, J. Diff. Geometry, 18 (1983), 279-315.

[2] S. K. Donaldson: The orientation of Yang-Mills moduli spaces and 4manifold topology, J. Diff. Geometry, 26(1987), 397-428.

[3] R. H. Fox and J. W. MILNOR: Singularities of 2-spheres in 4-space and cobordism of knots, Osaka J, Math., 3(1966), 257-267.

[4] Y. HIRAl: Representing homology classes of connected sums of 2-sphere bundles over 2-spheres, Kobe J. Math., 6(1989), 233-240.

[5] L. H. Kuaffman: On knots, Ann. of Math. Studies, 115, Princeton Univ. Press, Princeton, N. J., (1987).

[6] M. A. Kervaire and J. W. Milnor: On 2-sphere in 4-manifolds, Proc. Nat. Acad. Sei. U.S.A., 47 (1961), 1651-1657.

[7] K. KIXUCHI: Representing positive homology classes of $C P^{2} \# 2 \overline{C^{22}}$ and $C P^{2}$ \# $3 C P^{2}$, to appear in Proc. Amer. Math. Soc.

[8] R. KIRBY: A calculus for framed links in $S^{3}$, Invent. Math. 45(1978), 35-56.

[9] R. KiRbY: Problems in low-dimensional manifold theory, Proc. Symp. Pure Math. 32, American Mathematical Society, Providence, Rhode Island (1978), 273-312.

[10] R. KIRBY: 4-manifold problems, Contemp. Math., 35(1984), 513-528.

[11] T. LAWSON: Representing homology classes of almost definite 4-manifolds, Michigan Math. J., 34(1987), 85-91.

[12] Y. MATHIEU: Sur des noeuds qui ne sont pas déteminés par leur complément et problémes de cirugie dans les variétés de dimension 3, These, L'Université de Provence, 1990.

[13] K. MIYAZAKI: private communication.

[14] K. MOTEGI: Primeness of twisted knots, prepint.

[15] R. A. NORMAN: Dehn's Lemma for certain 4-manifolds, Invent. Math., $7(1969), 143-147$.

[16] V.A. ROHI,IN: Two-dimensional submanifolds of four-dimensional manifolds, Functional Anal. Appl., 5(1974), 39-48.

[17] S. SuzUk1: Local knots of 2-spheres in 4-manifolds, Proc. Japan Acad., 45 (1969), 34-38.

[18] S. H. WEINTRAUB: Inefficiently embedded surfaces in 4-manifolds, Algebraic Topology Aarhus 1978 (J. L. Dupont and I. H. Madsen, eds.), Lecture Notes in Math., 763, Springer-Verlag, Berlin and New York, 1979.

[19] M. YAMAMOTO: Lower bounds for the unknotting numbers of certain torus knots, Proc. Amer. Math. Soc., 86(1982), 519-524. 
[20] A. Yasuhara: $(2,15)$-torus knot is not slice in $C P^{2}$, Proc, Japan Acad. ser. A. Math. Sci., 67 (1991), 353-355.

Department of Mathematics

School of Science and Engineering

Waseda University

Tokyo 169-50

JAPAN

Recibido: 18 de marto de 1992 